\title{
Anomalous Hall effect and the role of Berry curvature in $\mathrm{Co}_{2}$ TiSn Heusler films
}

\author{
Benedikt Ernst, ${ }^{1}$ Roshnee Sahoo, ${ }^{1, *}$ Yan Sun, ${ }^{1}$ Jayita Nayak,,${ }^{1}$ Lukas Müchler, ${ }^{1}$ Ajaya K. Nayak, ${ }^{2}$ Nitesh Kumar, ${ }^{1}$ \\ Jacob Gayles, ${ }^{1}$ Anastasios Markou, ${ }^{1}$ Gerhard H. Fecher, ${ }^{1}$ and Claudia Felser ${ }^{1}$ \\ ${ }^{1}$ Max Planck Institute for Chemical Physics of Solids, Nöthnitzer Strasse 40, D-01187 Dresden, Germany \\ ${ }^{2}$ National Institute of Science Education and Research, Jatni, Bhubaneswar 752050, India
}

(Received 13 July 2017; revised manuscript received 13 June 2019; published 28 August 2019)

\begin{abstract}
Various $\mathrm{Co}_{2}$-based Heusler compounds are predicted to be half-metallic ferromagnets with Weyl points. These systems with lack of time inversion symmetry possess a momentum space Berry curvature that can introduce exotic transport properties. The present study, on epitaxially grown $\mathrm{Co}_{2} \mathrm{TiSn}$ films, is an approach to understand and explore this possibility. The theoretical investigation shows that the Berry curvature is significant for the total anomalous Hall effect in experimentally grown $\mathrm{Co}_{2} \mathrm{TiSn}$ films. The deviation between the theory and experiment is due to the influence of side jump and skew scattering mechanisms. From a theoretical point of view, the intrinsic contribution to the anomalous Hall effect originates from partially gaped nodal lines due to the symmetry reduction induced by the lack of time reversal symmetry. Furthermore, from hard x-ray photoelectron spectroscopy measurements, we establish an electronic structure in the film that is comparable to the theoretical density of states calculations. These results provide intuitive insight into Heusler spintronics rooted in topological electronic structure.
\end{abstract}

DOI: 10.1103/PhysRevB.100.054445

\section{INTRODUCTION}

Recently, spintronics has fascinated researchers, as it has abundant advantages over conventional electronics [1,2]. Heusler alloys have attracted interest in this regard due to their high Curie temperature $T_{\mathrm{C}}$ and tunability in terms of structural, electronic, and magnetic properties [3-7]. $\mathrm{Co}_{2-}$ based Heusler alloys have captivated unique attention, with the possibility to be half-metallic ferromagnets to utilize the full spin polarization at the Fermi energy [6,8,9]. A few of these materials were successfully fabricated into multilayered structures that exhibit giant magnetoresistance (GMR) and tunnel magnetoresistance (TMR) for nonvolatile memory applications [10,11]. A GMR ratio of $74.8 \%$ was reported by Sato et al. in a junction consisting of $\mathrm{Co}_{2} \mathrm{FeMnSi}$-based Heusler alloys [10]. Similarly, a large TMR ratio of $386 \%$ was observed at room temperature using $\mathrm{Co}_{2} \mathrm{FeAlSi}$ [11]. Recently, a remarkable TMR ratio of $2000 \%$ at $4.2 \mathrm{~K}(354 \%$ at $300 \mathrm{~K}$ ) was achieved using epitaxial $\mathrm{Co}_{2} \mathrm{MnSi}$ magnetic tunnel junctions [12]. The $\mathrm{Co}_{2} \mathrm{TiSn}$ compound is also one of the promising candidates that may be beneficial for spin manipulation due to its high Curie temperature and half-metallic properties $[13,14]$. Bulk $\mathrm{Co}_{2} \mathrm{TiSn}$ has been studied vividly in terms of electronic and magnetic properties [14-16]. For spintronics application as well as for a fundamental understanding, it is essential to implement such systems into thin films.

\footnotetext{
*sahoo@cpfs.mpg.de; sahoo.roshnee@gmail.com; Binayak Acharya Degree College, Berhampur-760006, Odisha, India

Published by the American Physical Society under the terms of the Creative Commons Attribution 4.0 International license. Further distribution of this work must maintain attribution to the author(s) and the published article's title, journal citation, and DOI.
}

The fundamental physics of the Berry curvature is intriguing as it helps us to understand intrinsic or dissipationless Hall currents in systems that are so useful to spintronics technology. There are many theoretical and experimental studies that aim to understand the fundamental origins of the anomalous Hall effect from the perspective of Berry curvature [17]. In recent studies, the anomalous Hall effect was reported in the noncollinear antiferromagnets $\mathrm{Mn}_{3} \mathrm{Ge}$ and $\mathrm{Mn}_{3} \mathrm{Sn}[18,19]$. The unconventional anomalous Hall effect in materials with a net-zero magnetic moment is understood in terms of a nonvanishing Berry curvature $[18,19]$. Recently, $\mathrm{Co}_{2}$-based Heusler compounds were predicted to be half-metallic ferromagnets with Weyl points $[19,20]$. The time-reversal symmetry breaking in these materials brings fascinating transport properties due to a large Berry phase [19-22]. $\mathrm{Co}_{2}$-based Heusler compounds were proposed to be half-metallic ferromagnets, where the band crossing points have been termed "magnetic monopoles" or Weyl points [20,21]. These band crossing points near the Fermi energy may lead to exotic transport phenomena [20,21].

In this paper, we focus on $\mathrm{Co}_{2} \mathrm{TiSn}$ films to understand the role of the Berry curvature with respect to the scattering mechanisms of the anomalous Hall effect. We find that the intrinsic anomalous Hall effect is theoretically due to partially gapped nodal lines. We use X-ray diffraction (XRD) and hard X-ray photoelectron spectroscopy (HAXPES) to analyze the structure and composition of the films. The total anomalous Hall effect shows contributions from both scattering mechanisms (skew scattering and side jump) and the intrinsic mechanism.

\section{THEORETICAL AND EXPERIMENTAL DETAILS}

We have calculated the electronic band structure by using the Vienna Ab initio Simulation Package (VASP) $[23,24]$. The 

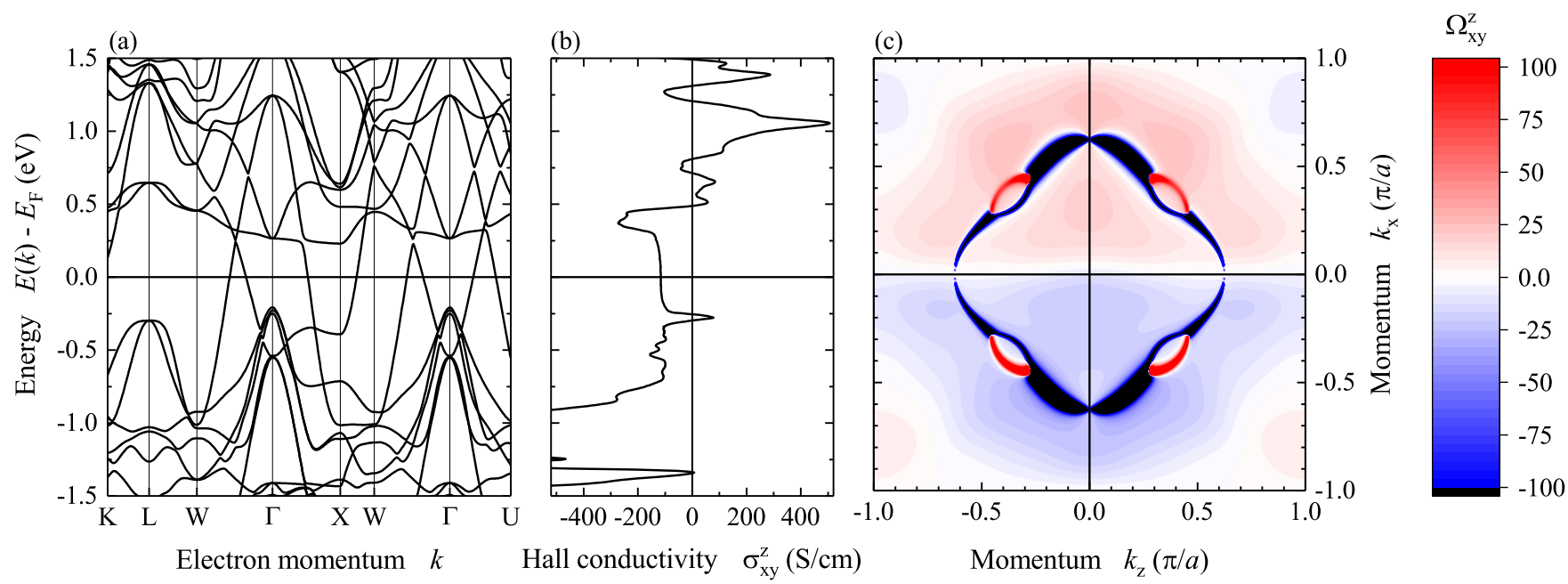

FIG. 1. (a) Electronic band structure along high-symmetry lines. (b) Energy-dependent anomalous Hall conductivity $\sigma_{x y}^{z}$. (c) Berry curvature $\Omega_{x y}^{z}$ distribution in the $k_{y}=0$ plane. The color bar is in arbitrary units.

exchange-correlation functional was considered in the generalized gradient approximation [25]. In all calculations, the experimental lattice parameters have been used. The magnetization was set along (001). In order to calculate the intrinsic anomalous Hall conductivity, we have projected the Bloch wave functions into maximally localized Wannier functions [26-28]. Based on the tight-binding model Hamiltonian, the anomalous Hall conductivity was calculated by the Kubo formula approach in the clean limit [17]:

$$
\begin{aligned}
\sigma_{x y}^{z} & =e^{2} \hbar\left(\frac{1}{2 \pi}\right)^{3} \int d \vec{k} \sum_{E(n, \vec{k})<E_{F}} f(n, \vec{k}) \Omega_{n, x y}^{z}(\vec{k}), \\
\Omega_{n, x y}^{z}(\vec{k}) & =2 \operatorname{Im} \sum_{n^{\prime} \neq n} \frac{\left\langle n, \vec{k}\left|\hat{v}_{x}\right| n^{\prime}, \vec{k}\right\rangle\left\langle n^{\prime}, \vec{k}\left|\hat{v}_{y}\right| n, \vec{k}\right\rangle}{\left[E(n, \vec{k})-E\left(n^{\prime}, \vec{k}\right)\right]^{2}},
\end{aligned}
$$

where $f(n, \vec{k})$ is the Fermi-Dirac distribution, $E(n, \vec{k})$ is the eigenvalue of the $n$th eigenstate of $|u(n, \vec{k})\rangle$ at $\vec{k}$ points, and $v_{\alpha}=\frac{1}{\hbar} \frac{\partial H(\vec{k})}{\partial k_{\alpha}}$ is the velocity operator. A $500 \times 500 \times 500$ grid of $k$ points was used in the evaluation of the integrals.

Thin films of $\mathrm{Co}_{2}$ TiSn were grown on polished $\mathrm{MgO}(001)$ substrates by cosputtering from elementary $\mathrm{Co}, \mathrm{Ti}$, and $\mathrm{Sn}$ targets using an ultrahigh vacuum BESTEC sputtering system. These films are named according to their growth temperatures such as CTS-550, CTS-600, and CTS-650, grown at $550^{\circ} \mathrm{C}$, $600^{\circ} \mathrm{C}$, and $650^{\circ} \mathrm{C}$, respectively. The CTS-650 film was annealed for $30 \mathrm{~min}$ at $450^{\circ} \mathrm{C}$ and named CTS-650 + A. All films were capped with $2-3 \mathrm{~nm}$ of aluminum at room temperature to prevent oxidation. The stoichiometry of the films was checked with energy dispersive $\mathrm{x}$-ray spectroscopy (EDX). The crystal structure and thickness of the films were determined using a standard Pananalytical x-ray diffractometer using $\mathrm{Cu} K \alpha$ radiation. Magnetic properties were measured in a Quantum Design magnetometer (MPMS 3). The transport properties were performed in a Quantum Design physical properties measurement system. HAXPES on the $\mathrm{Co}_{2} \mathrm{TiSn}$ thin films was performed at beamline BL47XU of Spring-8 (Japan). The electron energy distribution was recorded by a hemispherical energy analyzer (R4000-HV, Scienta). The photon energy was fixed at $h v=7.94 \mathrm{keV}$ using a doublecrystal monochromator [Si(111)] with a postmonochromator $[\mathrm{Si}(444)]$. The overall energy resolution, including the monochromator and analyzer resolution, was determined by fitting the Au Fermi edge and was found to be $250 \mathrm{meV}$ at $300 \mathrm{~K}$. The experiment was carried out in near-normal emission geometry (see Refs. [29,30] for more details of the HAXPES experiments).

\section{RESULTS AND DISCUSSION}

\section{A. Berry curvature calculation}

We have calculated the Berry curvature for $\mathrm{Co}_{2} \mathrm{TiSn}$ as presented in Fig. 1. $\mathrm{Co}_{2} \mathrm{TiSn}$ exhibits the fcc $L 2_{1}\left(\mathrm{Cu}_{2} \mathrm{MnAl}\right.$, $c F 16,225)$ structure. In the absence of any net magnetic moment, $\mathrm{Co}_{2} \mathrm{TiSn}$ possesses three mirror planes, $m_{x}, m_{y}$, and $m_{z}$, which protect the gapless nodal-line-like band structure in the $k_{x}=0, k_{y}=0$, and $k_{z}=0$ planes, respectively [21,31]. If the magnetic moment is considered, the symmetry is reduced. For example, as performed in our experiments, if we apply a magnetic field along the $z$ direction, then the mirrors $m_{x}$ and $m_{y}$ are no longer symmetry planes, while $m_{z}$ is still a symmetry plane since the $z$ component of the spin $S_{z}$ is left invariant by $m_{z}$. Therefore, the gapless nodal line exists only in the $k_{z}=0$ plane, and hence, the nodal lines in the $k_{x}=0$ and $k_{y}=0$ planes exhibit a finite band gap due the magnetic moment oriented in the $z$ direction. Additionally, two Weyl points with a topological charge of \pm 2 emerge along $k_{z}$ that derive from the nodal line $[21,31]$. The intrinsic contribution of Weyl fermions to the anomalous Hall conductivity (AHC) is proportional to their $k$ space distance along the magnetic field direction. However, we do not observe a direct contribution of these points from the band structure calculations. This might be explained by the fact that the Weyl points are several hundred meV above the Fermi energy and due to the presence of other Weyl points in planes of constant $k_{z}$ [31].

The gapless nodal line band structure contains nonzero Berry curvatures around it; however, they are helically distributed in the mirror plane, and the total flux is zero. Hence, 


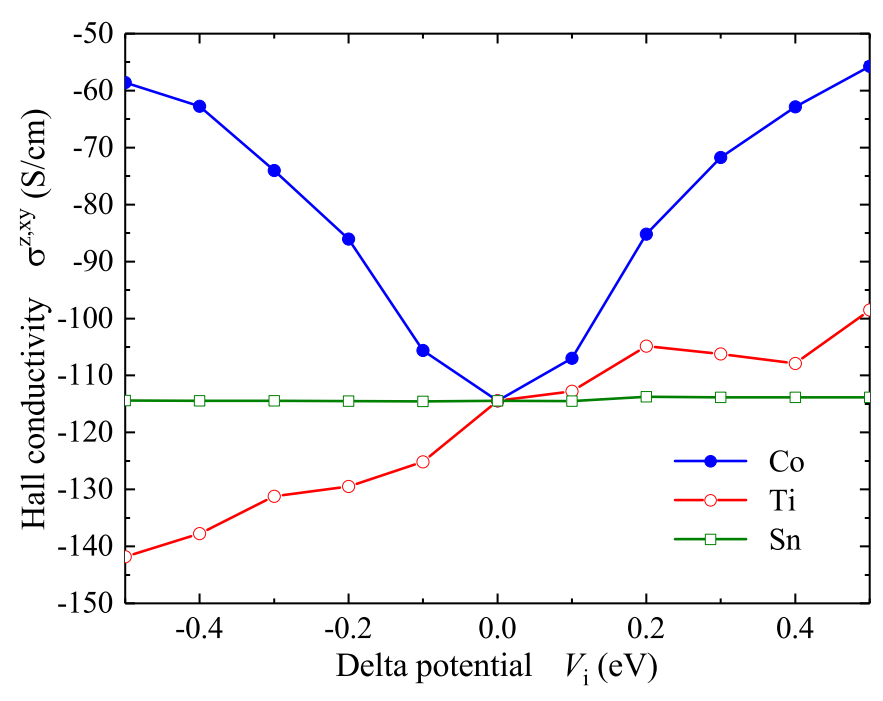

FIG. 2. Anomalous Hall conductivity as a function of the $\delta$ potentials at different atomic sites in $\mathrm{Co}_{2} \mathrm{TiSn}$.

the gapless nodal line in the $k_{z}=0$ plane does not contribute to the intrinsic anomalous Hall conductivity. On the other hand, the nodal lines in the $k_{x}=0$ and $k_{y}=0$ planes are gapped by the magnetization, which also forces the Berry curvature to orient along the direction of magnetization. Taking the $k_{y}=0$ plane as an example, as presented in Fig. 1(c), the $k$ points around the broken nodal lines are dominated by negative $\Omega_{x y}^{z}$ components of the Berry curvature and directly contribute to the negative anomalous Hall effect, which has the same mechanism as the strong spin Hall effect in nodal line materials $[32,33]$. The nodal lines in mirror planes have strong energy dispersion [21,31], and therefore, only part of the nodal lines contribute to the anomalous Hall conductivity at fixed Fermi level. The intrinsic anomalous Hall conductivity is almost constant at $\sigma_{H}=-\sigma_{x y}^{z}=100 \mathrm{~S} / \mathrm{cm}$ in the range of -0.25 to $0.25 \mathrm{eV}$, which is just inside the energy window of the dispersion of the nodal lines [see Figs. 1(a) and 1(b)]. Therefore, the intrinsic anomalous Hall conductivity in $\mathrm{Co}_{2}$ TiSn mainly originates from the magnetization-induced gaps by breaking the nodal line band structure.

\section{B. Disorder effect on anomalous Hall conductivity}

In order to understand the effect of structural disorder on the measured anomalous Hall effect of the material, we performed additional calculations. In these calculations, we used the perturbation approximation where we introduce a local $\delta$ potential to different sites:

$$
H=H_{0}+V_{i} \delta\left(r-r_{i}\right) .
$$

Here, $r_{i}$ is the position of atom $i$. The disorder strength is proportional to the $\delta$ potential. From the simulation (Fig. 2), it is found that the disorder at the Ti sites and, especially, the Co sites has a strong influence on the intrinsic anomalous Hall effect, whereas the effect of disorder on Sn sites is negligible. This is explainable, as the bands in the vicinity of the Fermi energy and the magnetic moments are mainly contributed by $\mathrm{Co}$ and $\mathrm{Ti}$ atoms. The disorder at Co sites (symmetriclike) decreases the intrinsic anomalous Hall conductivity independent of the sign of the $\delta$ potential, whereas the disorder at Ti sites (antisymmetriclike) increases the intrinsic anomalous Hall conductivity when the local potential is negative.

The absolute value of the calculated Hall conductivity in the vicinity of the band crossing at about $0.25 \mathrm{eV}$ above the Fermi energy is about $250 \mathrm{~S} / \mathrm{cm}$, which is 2.5 times higher than the value obtained near the Fermi energy (see Fig. 1). To reach this band filling one needs doping by 0.2 electron. This may be achieved, for example, by some Co excess in $\mathrm{Co}_{2} \mathrm{TiSn}$. When assuming a distribution of the excess Co on Ti sites or Sn sites, the composition might become $\mathrm{Co}_{2.044} \mathrm{Ti}_{0.956} \mathrm{Sn}$ or $\mathrm{Co}_{2.044} \mathrm{TiSn}_{0.956}$. It is important to note here that such a small addition of $\mathrm{Co}$ and the accompanied chemical disorder will be too small to detect by x-ray diffraction. This explains how disorder in the form of a slightly off-stoichiometric composition can influence the value of the intrinsic anomalous Hall conductivity.

\section{Structural properties of the thin films}

In this and the next sections, we focus on the experimental results, where we begin with the crystal structure of the epitaxial $\mathrm{Co}_{2} \mathrm{TiSn}$ films grown under different conditions as mentioned in Sec. II. Figure 3(a) depicts the $\theta-2 \theta$ XRD pattern for various $\mathrm{Co}_{2} \mathrm{TiSn}$ films. All films exhibit (002) and (004) peaks of the cubic $L 2_{1}$ Heusler structure along with the (002) peak of the $\mathrm{MgO}$ substrate. The $L 2_{1}$ crystal structure of Heusler compounds is presented in the inset of Fig. 3(a). In this structure, $\mathrm{Co}, \mathrm{Ti}$, and $\mathrm{Sn}$ atoms occupy Wyckoff positions $8 \mathrm{c}, 4 \mathrm{~b}$, and $4 \mathrm{a}$, respectively.

The cubic lattice parameter of CTS-550 is found to be $a=6.02 \AA$. The shift of the XRD peaks towards lower $2 \theta$ value points to an increase of the lattice parameter with higher growth temperatures. The lattice parameter for CTS-650 + A is $6.1 \AA$. The epitaxial relationship of the films with the substrate is found to be [100] $\mathrm{Co}_{2}$ TiSn \| [110] MgO. X-ray reflectivity (XRR) was measured to determine the thickness and surface smoothness of the film. The XRR measurement for CTS-650 + A is shown in Fig. 3(b). The clear oscillation of fringes suggests that the surface of this film is smooth. Upon fitting the XRR curve, the thickness and roughness are estimated to be 22 and $0.3 \mathrm{~nm}$, respectively. The $\phi$ scan is performed along the (111) plane. It is known that the sample with $L 2_{1}$ structure produces a characteristic (111) diffraction peak [34]. From $\phi$ scans [as given in the inset of Fig. 3(b)], it is seen that the superlattice (111) peak has the highest integrated intensity values for CTS-650 + A compared to CTS-550 and CTS-650. This confirms that CTS-650 + A possesses the highest degree of $L 2_{1}$ order for all of the investigated samples. Furthermore, EDX analysis confirms that the Co:Ti:Sn stoichiometry of the investigated films is 2:1:1 within an uncertainty of $5 \%$.

\section{Hard x-ray photoelectron spectroscopy}

In addition to $\mathrm{x}$-ray diffraction and reflectivity measurements, the films were further investigated with respect to their quality by HAXPES. HAXPES is a powerful technique that is utilized to understand the detailed electronic structure of various types of materials $[29,30,35]$. It helps to investigate 

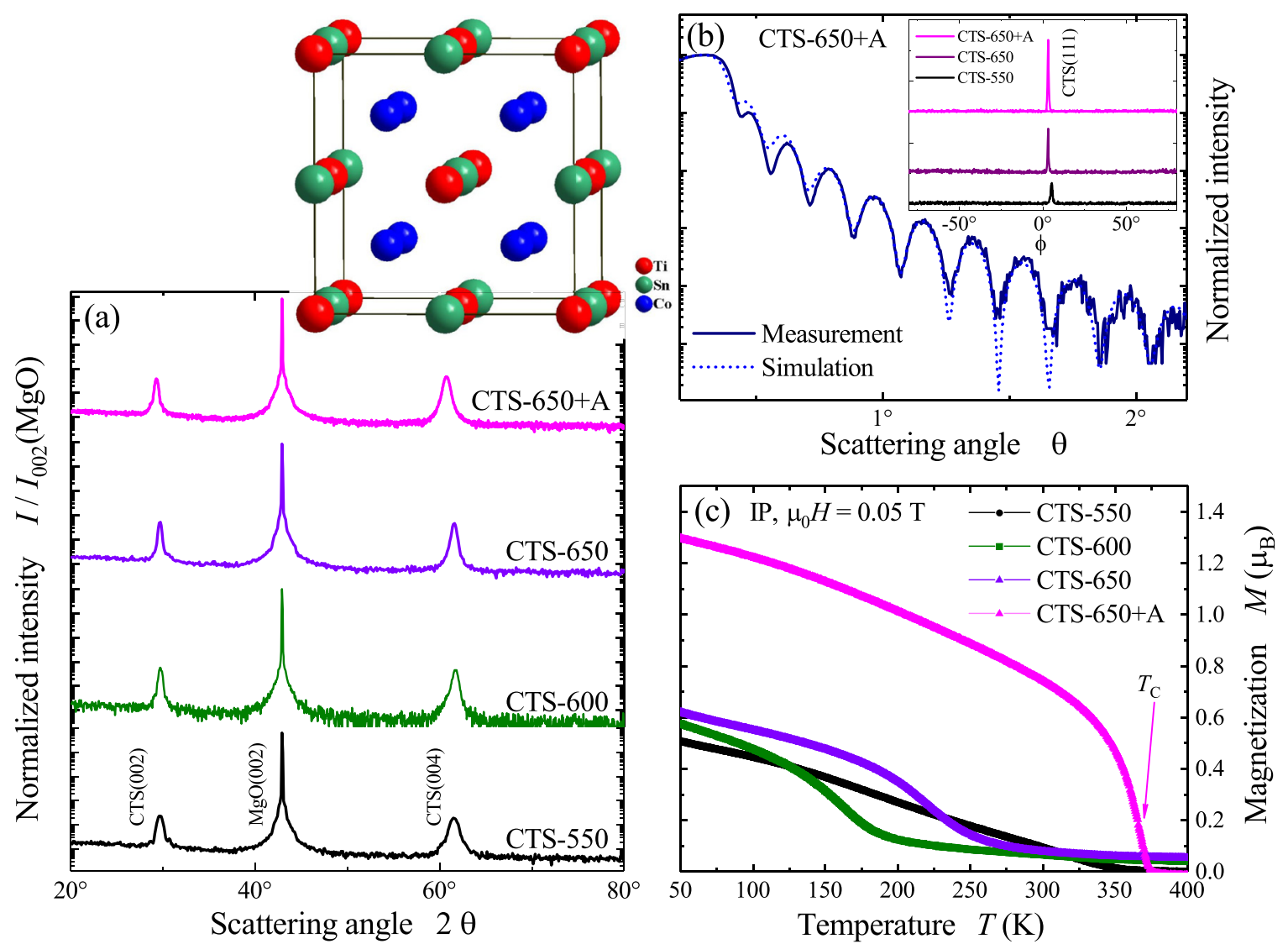

FIG. 3. (a) $\theta-2 \theta$ XRD diffraction data recorded at room temperature for different $\mathrm{Co}_{2}$ TiSn films. The inset shows the cubic $L 2_{1}$ crystal structure of Heusler compounds. (b) XRR data for CTS-650 + A. The inset shows azimuthal $(\phi)$ scans of the (111) superlattice reflex for different $\mathrm{Co}_{2}$ TiSn films. (c) Temperature dependence of the in-plane (IP) magnetization of the $\mathrm{Co}_{2} \mathrm{TiSn}$ films measured at $0.05 \mathrm{~T}$.

the bulk electronic structure, as was explained in detail for Heusler compounds [36,37]. The high-kinetic-energy electrons exhibit a large probing depth inside the material and provide bulk properties that are free of surface effects. In particular, the details of the valence band density of states are probed due to the integration over a large volume in momentum space at high kinetic energies. Therefore, the HAXPES valence band spectrum [see Fig. 4(b)] has been measured for the well-ordered CTS-650 + A and is compared to the calculated density of states, as shown in Fig. 4(a). In the valence band spectrum the high intensity at $-9.3 \mathrm{eV}$ arises from the contribution of $s$ electrons that are mainly localized close to the $\mathrm{Sn}$ atoms, as seen from the calculations. On the other hand, a minimum appears at about $-7.3 \mathrm{eV}$ that reflects the $s p$ hybridization gap in the density of states and is typical for Heusler compounds. In the upper part of the spectrum, four maxima emerge at $-5.8,-3.5,-2.0$, and $-0.4 \mathrm{eV}$ binding energies. The feature at $-3.5 \mathrm{eV}$ arises from the $p d$ hybridization of Co $d$ states with Sn $p$ states. Closer to the Fermi energy, the spectra are dominated by $d$ states localized mainly at the Co atoms. The valence band spectrum thus reflects the most dominant features of the calculated density of states, which accounts for matrix elements that redistribute the intensities compared to the densities.

Core level spectra are used to explain the cleanness of the compound and, with restrictions, its electronic state, chemical order, and composition. The Co $2 p$ core level spectrum
[Fig. 4(c)] exhibits a distinct spin-orbit splitting of $14.9 \mathrm{eV}$. A similar size of the spin-orbit splitting $(14.95 \mathrm{eV})$ was also reported for polycrystalline samples [14]. No oxide-related features at $2-3 \mathrm{eV}$ higher binding energy compared to the Co main peak are detected. The Sn $3 p$ core level spectrum
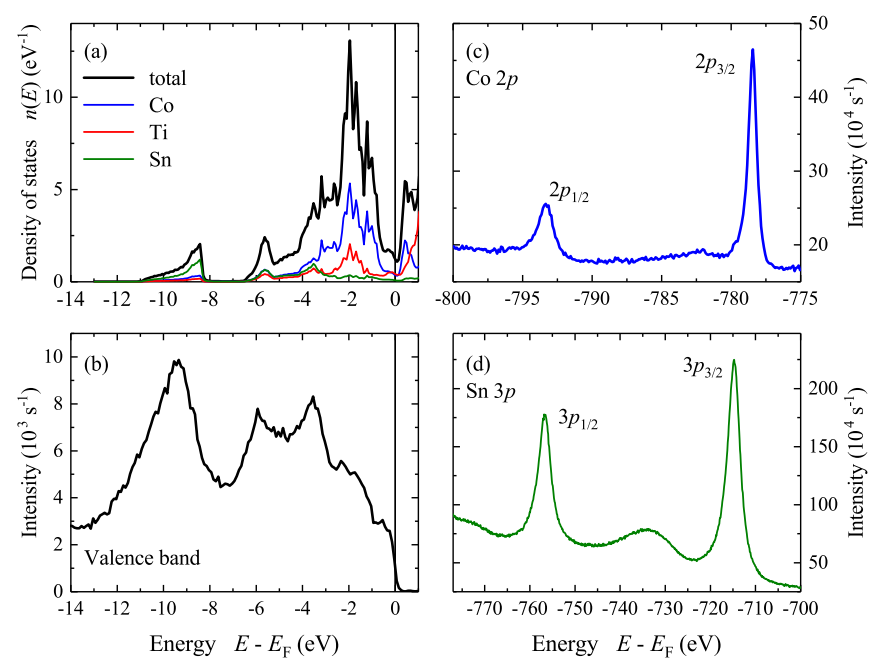

FIG. 4. (a) Calculated density of states of $\mathrm{Co}_{2} \mathrm{TiSn}$. (b) Hard $\mathrm{x}-$ ray valence band spectrum. (c) Co $2 p$ and (d) Sn $3 p$ core level spectra of CTS-650 + A measured by employing hard x rays $(7.9 \mathrm{keV})$. 

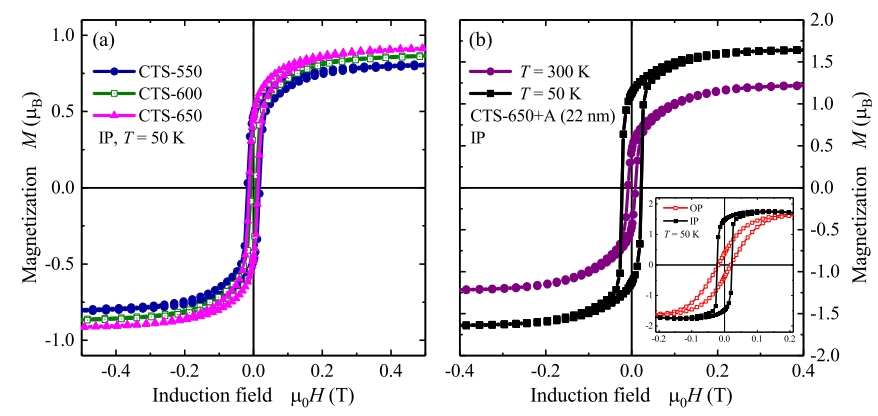

FIG. 5. Isothermal magnetization curves of (a) CTS-550, CTS600, and CTS-650 at $50 \mathrm{~K}$ and (b) CTS-650 + A at 50 and $300 \mathrm{~K}$. The inset shows in-plane (IP) and out-of-plane (OP) curves of CTS-650 + A at $50 \mathrm{~K}$.

exhibits a large spin-orbit splitting of about $42 \mathrm{eV}$ [Fig. 4(d)] together with pronounced side maxima. These broad transitions emerge not as primary excitations but as energy loss structures from secondary band-band or plasmon excitations. The latter result from the free-electron-type behavior of the $s$ electrons localized mostly close to Sn, as mentioned above. Again, no oxygen or other impurity-related features are detected. This indicates, together with the Co $2 p$ spectrum, the cleanness of the sample. In all cases, no significant chemical shifts of core level binding energies are found in $\mathrm{Co}_{2} \mathrm{TiSn}$. These signify the metallic character of the compound. All observed features are in qualitative agreement with the previous HAXPES measurement on polycrystalline bulk samples [14], which reflect the high quality of the thin films.

\section{E. Magnetic and electrical properties}

This section reports on the magnetic properties and Hall effect measurements of the epitaxially grown $\mathrm{Co}_{2} \mathrm{TiSn}$ thin films. Figure 3(c) shows the temperature-dependent magnetization curves $M(T)$ that are measured in the field-cooled mode in the presence of an in-plane field of 0.05 T. For CTS550 , the magnetic transition is quite broad in nature without a well-defined Curie temperature $T_{C}$. When the growth temperature is increased, CTS-600 and CTS-650 exhibit a welldefined $T_{C}$ of about 160 and $225 \mathrm{~K}$, respectively. However, the nonzero magnetization above $T_{C}$ for these films indicates the presence of some disorder. We found by annealing of CTS-650 that a very sharp $T_{C}$ of $\sim 366 \mathrm{~K}$ is obtained for the CTS-650 + A film. For bulk $\mathrm{Co}_{2} \mathrm{TiSn}$, a $T_{C}$ of $355 \mathrm{~K}$ was reported by Barth et al. [14]. It should be mentioned here that the trend of $T_{C}$ in the observed films is an important factor to say about the degree of order or disorder in the system. The observation of undefined or low $T_{C}$ in CTS-550, CTS-600, and CTS-650 suggests the presence of large chemical disorder. For instance, Meinert et al. [13] have reported a considerable decrease in atomic disorder with the increase of deposition temperature. The presence of Co-Ti antisite disorder, as found by NMR and Mößbauer measurements, has also been reported for bulk [6]. Therefore, disorder plays an important role in the present system. It can be reduced considerably with higher substrate temperatures during growth.

In-plane magnetic hysteresis loops $M(H)$ for different $\mathrm{Co}_{2}$ TiSn films measured at $50 \mathrm{~K}$ are presented in Fig. 5(a).
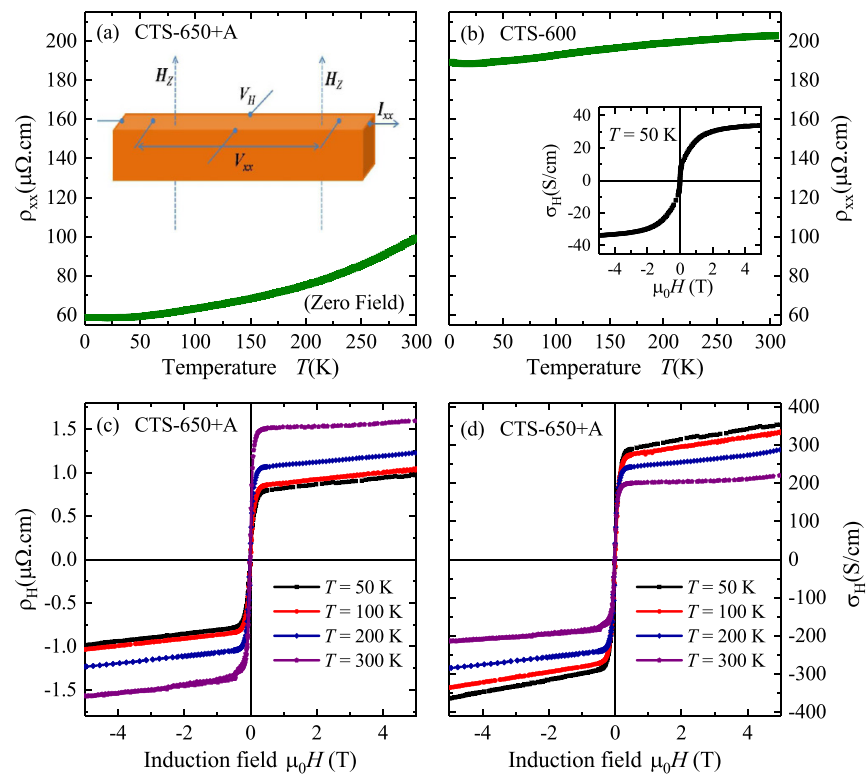

FIG. 6. Temperature dependence of the linear electrical resistivity $\rho_{x x}$ (a) for CTS-650 + A and (b) for CTS-600. The inset of (a) shows a schematic diagram for the measurement of linear voltage $V_{x x}$ and lateral voltage $V_{H}$. The inset of (b) shows the field dependence of Hall conductivity at $50 \mathrm{~K}$ for CTS-600. (c) Field dependence of Hall resistivity at different temperatures for CTS-650 + A. (d) Field-dependent Hall conductivity at various temperatures for CTS-650 + A.

The saturation magnetic moment per formula unit increases with the increase in growth temperature from $0.8 \mu_{B}$ for CTS550 to $0.9 \mu_{B}$ for the CTS- 650 . For CTS- $650+\mathrm{A}$ the saturated moment further increases to $1.6 \mu_{B}$ at $50 \mathrm{~K}$ and $1.2 \mu_{B}$ at $300 \mathrm{~K}$ [Fig. 5(b)]. The presence of an in-plane easy axis is confirmed by the measurement of out-of-plane $M(H)$ loops that exhibit a hard-axis-type hysteresis [inset of Fig. 5(b)] in the present film. As compared to all other films, CTS-650 + A possesses a larger magnetic moment and higher $T_{C}$.

To characterize the anomalous Hall effect in the present thin films, we carried out detailed electrical transport measurements. A schematic diagram of the present measurements is illustrated in the inset of Fig. 6(a). In the given configuration, the current $I_{x x}$ is applied along [100], the magnetic field $H_{z}$ is applied along [001], and the lateral Hall voltage $V_{H}$ is measured along [010]. As shown in Fig. 6(a), the temperature dependence of the linear resistivity $\rho_{x x}$ measured at zero field exhibits a metallic characteristic. $\rho_{x x}$ values of 58 and $100 \mu \Omega \mathrm{cm}$ are obtained at temperatures of 2 and $300 \mathrm{~K}$, respectively. Although CTS-600 displays a metallic behavior in the resistivity measurement, the magnitude of resistivity is substantially higher than that of CTS-650 + A. The higher residual resistivity of CTS-600 clearly indicates the presence of a higher degree of disorder, as discussed earlier. The residual resistivity ratio (RRR) is calculated from the resistivities at low $(2 \mathrm{~K})$ and high $(300 \mathrm{~K})$ temperatures: $\mathrm{RRR}=\rho_{x x}(300 \mathrm{~K}) / \rho_{x x}(2 \mathrm{~K})$. For CTS-650 + A, we find a RRR value of 1.7 , whereas it is only 1.07 for CTS-600. The lower RRR value implies higher impurity scattering due to a 
larger degree of disorder in CTS-600. Therefore, hereafter we focus on sample CTS-650 + A.

The Hall resistivity $\rho_{H}$ is obtained from the transverse Hall voltage $V_{H}$, as shown in the schematic diagram in the inset of Fig. 6(a). In a ferromagnet, $\rho_{H}$ is usually written as

$$
\rho_{H}=\mu_{0}\left(R_{H} H+R_{M} M\right),
$$

where $R_{H}$ and $R_{M}$ are the normal and anomalous Hall coefficients, $\mu_{0}$ is the vacuum permeability, and $H$ and $M$ are the magnetic field and the magnetization, respectively. The first term is the ordinary Hall resistivity that arises from the Lorentz force, and the second term illustrates the anomalous Hall contribution that originates from the intrinsic magnetization. Figure 6(c) presents $\rho_{H}$ versus applied field for the film CTS-650 + A. $\rho_{H}$ initially increases with field before it tends towards the saturation. A weak linear increase in $\rho_{H}$ at higher field emerges from the contribution of the ordinary Hall effect. Contrary to the magnetic behavior, where larger magnetization is observed at lower temperature, $\rho_{H}$ of $1 \mu \Omega \mathrm{cm}$ is obtained at $50 \mathrm{~K}$ and increases to $1.5 \mu \Omega \mathrm{cm}$ at $300 \mathrm{~K}$. The Hall conductivity $\sigma_{H}$ has been extracted with the formula

$$
\sigma_{H}=\frac{\rho_{H}}{\left[\rho_{x x}^{2}+\rho_{H}^{2}\right]} .
$$

The field dependence of the Hall conductivity $\sigma_{H}$ is plotted in Fig. 6(d) (note that $\sigma_{H}=-\sigma_{x y}^{z}$ when comparing experimental and theoretical values). $\sigma_{H}$ increases rapidly under a relatively small field due to the anomalous contribution. This anomalous contribution has values of 284 and $180 \mathrm{~S} / \mathrm{cm}$ at 50 and $300 \mathrm{~K}$, respectively. In contrast, CTS-600 shows a Hall conductivity of about $35 \mathrm{~S} / \mathrm{cm}$ at $50 \mathrm{~K}$ [inset of Fig. 6(b)]. The Hall coefficient $R_{H}$ for CTS-650 + A is obtained from the slope of $\rho_{H}$ versus $H$. It is observed that the Hall coefficient is positive for all temperatures, which suggests that the charge carriers are of the hole type or, correspondingly, the electrons have a negative effective mass. The carrier concentration $n=$ $e / R_{H}$ is calculated from the Hall constant $R_{H}$, where $e$ is the electron charge. The carrier concentration is calculated to be $1.57 \times 10^{22} \mathrm{~cm}^{-3}$ at $300 \mathrm{~K}$, which corresponds to a metallic kind of behavior. The carrier concentration value decreases linearly with a decrease in temperature, and for $2 \mathrm{~K}$ it is obtained as $1.41 \times 10^{22} \mathrm{~cm}^{-3}$.

Further, we discuss the physical origin of the anomalous Hall effect in CTS-650 + A. From the Berry curvature calculations, it is found that an anomalous Hall conductivity of $100 \mathrm{~S} / \mathrm{cm}$ is attained in $\mathrm{Co}_{2} \mathrm{TiSn}$, whereas the measurement gives an anomalous Hall conductivity of $\sim 284 \mathrm{~S} / \mathrm{cm}$. In order to understand the difference, it is necessary to find out whether there is a contribution from any extrinsic mechanisms, such as skew scattering or side jumps $[38,39]$. We can separate the extrinsiclike and intrinsiclike contributions from the anomalous Hall conductivity values with the scaling of the linear resistivity. The anomalous Hall effect can be written in terms of extrinsic or intrinsic effects as [17]

$$
\begin{gathered}
\rho_{H}=\left[\alpha \rho_{x x}+(\beta+b) \rho_{x x}^{2}\right] M, \\
\frac{\rho_{H}}{\rho_{x x} M}=\alpha+(\beta+b) \rho_{x x} .
\end{gathered}
$$

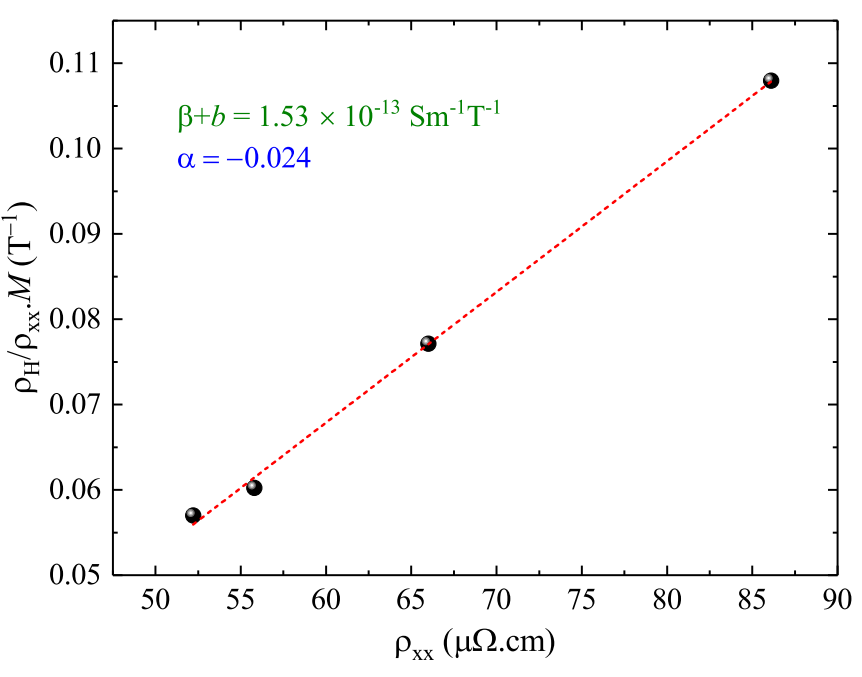

FIG. 7. Experimental data (black spheres) are plotted as $\frac{\rho_{H}}{\rho_{x x} M}$ vs $\rho_{x x}$ for CTS-650 $+\mathrm{A}$. The linear fit is shown as a red dashed line.

Here, the terms $\alpha$ and $\beta$ correspond to skew scattering and side jump mechanisms, which are extrinsic in nature, whereas the term $b$ corresponds to the intrinsic Berry curvature. $M$ in the given equation specifies the saturation magnetization. In Fig. 7 we plot $\frac{\rho_{H}}{\rho_{r x} M}$ extracted from different temperatures as a function of $\rho_{x x}$. The intercept and slope correspond to $\alpha$ and $(\beta+b)$, which are determined from a linear fit. The values are given in Fig. 7. Here, the anomalous Hall conductivity can be separated into skew scattering and side jump + intrinsic, from the total anomalous Hall conductivity. The anomalous Hall conductivities arising from skew scattering are obtained as -104 and $-36 \mathrm{~S} / \mathrm{cm}$ at 50 and $300 \mathrm{~K}$, respectively, from the intercept parameter $\alpha$. Similarly, for $\beta+b$, the anomalous Hall conductivity that arises from the combination of intrinsic and side jump mechanisms is calculated as 394 and $226 \mathrm{~S} / \mathrm{cm}$ at 50 and $300 \mathrm{~K}$, respectively. The net anomalous Hall conductivity agrees with the experimental values within an error of $\pm 15 \mathrm{~S} / \mathrm{cm}$. It is important to mention here that at $2 \mathrm{~K}$ a linear conductivity $\sigma_{x x}$ of $\sim 1.9 \times 10^{4} \mathrm{~S} / \mathrm{cm}$ is obtained for the given film. This value falls into the category of a goodmetal regime, where the anomalous Hall effect is considered to mainly arise from the intrinsic and side jump mechanism [17]. However, from the current analysis presented in Fig. 7, it is found that in addition to the intrinsic effect, skew scattering as well as side jumps also contribute to the anomalous Hall effect in the investigated film.

\section{SUMMARY}

In summary, Berry curvature calculations were performed for $\mathrm{Co}_{2} \mathrm{TiSn}$, and an intrinsic anomalous Hall conductivity of $100 \mathrm{~S} / \mathrm{cm}$ was calculated. This anomalous Hall conductivity originates from slightly gaped nodal lines due to a symmetry reduction induced by the magnetization. From the present analysis, it was also found that Weyl points exist a few hundred meV above the Fermi energy. The position of the Weyl nodes may be manipulated to appear at the Fermi energy by doping $\mathrm{Co}_{2} \mathrm{TiSn}$ to increase the valence electron 
concentration. From the perturbation approximation, it can also be seen that the atomic site disorder can also influence the AHC values. Moreover, the valence band HAXPES spectra measured for the $\mathrm{CO}_{2} \mathrm{TiSn}$ film suggested that the electronic structure in this film is in agreement with the calculated density of states. Experimentally, the film exhibited an anomalous Hall conductivity of 284 and $180 \mathrm{~S} / \mathrm{cm}$ at 50 and $300 \mathrm{~K}$, respectively. The larger value of the measured anomalous Hall conductivity is attributed to the discussed skew scattering and side jump mechanisms. The present work emphasizes the potential of $\mathrm{Co}_{2} \mathrm{TiSn}$ for future spintronics applications.

\section{ACKNOWLEDGMENTS}

The authors thank J. Kübler for useful discussions on theory, C. Shekhar for measuring transport properties, and E. Ikenaga for experimental support during the HAXPES measurements. R. Sahoo thanks the Department of Science and Technology (DST), government of India, for a DST Inspire Research Grant. The synchrotron radiation HAXPES experiments were performed at BL47XU of Spring-8 with approval of JASRI (Proposal No. 2016B1086). This work was financially supported by the ERC Advanced Grant (291472) "Idea Heusler."
[1] S. S. P. Parkin, X. Jiang, C. Kaiser, A. Panchula, K. Roche, and M. Samant, Proc. IEEE 91, 661 (2003).

[2] F. Pulizzi, Nat. Mater. 11, 367 (2012).

[3] Spintronics: From Materials to Devices, edited by C. Felser and G. H. Fecher (Springer, Dordrecht, 2013).

[4] T. Graf, C. Felser, and S. S. P. Parkin, Prog. Solid State Chem. 39, 1 (2011).

[5] J. Kübler, G. H. Fecher, and C. Felser, Phys. Rev. B. 76, 024414 (2007).

[6] H. C. Kandpal, G. H. Fecher, and C. Felser, J. Phys. D 40, 1507 (2007).

[7] R. Sahoo, L. Wollmann, S. Selle, T. Höche, B. Ernst, A. Kalache, C. Shekhar, N. Kumar, S. Chadov, C. Felser, S. S. P. Parkin, and A. K. Nayak, Adv. Mater. 28, 8499 (2016).

[8] J. Kübler, A. R. Williams, and C. B. Sommers, Phys. Rev. B. 28, 1745 (1983).

[9] I. Galanakis, P. H. Dederichs, and N. Papanikolaou, Phys. Rev. B. 66, 174429 (2002).

[10] J. Sato, M. Oogane, H. Naganuma, and Y. Ando, Appl. Phys. Express 4, 113005 (2011).

[11] N. Tezuka, N. Ikeda, F. Mitsuhashi, and S. Sugimoto, Appl. Phys. Lett. 94, 162504 (2009).

[12] H. Liu, Y. Honda, T. Taira, K. Matsuda, M. Arita, T. Uemura, and M. Yamamotoa, Appl. Phys. Lett. 101, 132418 (2012).

[13] M. Meinert, J. Schmalhorst, H. Wulfmeier, G. Reiss, E. Arenholz, T. Graf, and C. Felser, Phys. Rev. B. 83, 064412 (2011)

[14] J. Barth, G. H. Fecher, B. Balke, S. Ouardi, T. Graf, C. Felser, A. Shkabko, A. Weidenkaff, P. Klaer, H. J. Elmers et al., Phys. Rev. B. 81, 064404 (2010).

[15] H. C. Kandpal, V. Ksenofontov, M. Wojcik, R. Seshadri, and C. Felser, J. Phys. D 40, 1587 (2007).

[16] J. Barth, G. H. Fecher, B. Balke, T. Graf, A. Shkabko, A. Weidenkaff, P. Klaer, M. Kallmayer, H. J. Elmers, H. Yoshikawa, S. Ueda, K. Kobayashi, and C. Felser, Philos. Trans. R. Soc. A. 369, 3588 (2011).

[17] N. Nagaosa, J. Sinova, S. Onoda, A. H. MacDonald, and N. P. Ong, Rev. Mod. Phys. 82, 1539 (2010).

[18] A. K. Nayak, J. E. Fischer, Y. Sun, B. Yan, J. Karel, A. C. Komarek, C. Shekhar, N. Kumar, W. Schnelle, J. Kübler, C. Felser, and S. S. P. Parkin, Sci. Adv. 2, e1501870 (2016).
[19] S. Nakatsuji, N. Kiyohara, and T. Higo, Nature (London) 527, 212 (2015).

[20] J. Kübler and C. Felser, Europhys. Lett. 114, 47005 (2016).

[21] Z. Wang, M. G. Vergniory, S. Kushwaha, M. Hirschberger, E. V. Chulkov, A. Ernst, N. P. Ong, R. J. Cava, and B. A. Bernevig, Phys. Rev. Lett. 117, 236401 (2016).

[22] J. Kübler and C. Felser, Phys. Rev. B. 85, 012405 (2012).

[23] G. Kresse and J. Furthmüller, Phys. Rev. B 54, 11169 (1996).

[24] G. Kresse and J. Furthmüller, Comput. Mater. Sci. 6, 15 (1996).

[25] J. P. Perdew, K. Burke, and M. Ernzerhof, Phys. Rev. Lett. 77, 3865 (1996).

[26] N. Marzari and D. Vanderbilt, Phys. Rev. B 56, 12847 (1997).

[27] I. Souza, N. Marzari, and D. Vanderbilt, Phys. Rev. B 65, 035109 (2001).

[28] A. A. Mostofi, J. R. Yates, Y. S. Lee, I. Souza, D. Vanderbilt, and N. Marzari, Comput. Phys. Commun. 178, 685 (2008).

[29] S. Ouardi, G. H. Fecher, and C. Felser, J. Electron Spectrosc. Relat. Phenom. 190, 249 (2013).

[30] G. H. Fecher and C. Felser, Hard X-Ray Photoelectron Spectroscopy of New Materials for Spintronics (Springer, Dordrecht, 2013), Chap. 11, p. 243.

[31] G. Chang, S. Y. Xu, H. Zheng, B. Singh, C. H. Hsu, I. Belopolski, D. S. Sanchez, G. Bian, N. Alidoust, H. Lin, and M. Z. Hasan, Sci. Rep. 6, 38839 (2016).

[32] Y. Sun, Y. Zhang, C. Felser, and B. Yan, Phy. Rev. Lett. 117, 146403 (2016).

[33] Y. Sun, Y. Zhang, C. X. Liu, C. Felser, and B. Yan, Phys. Rev. B. 95, 235104 (2017).

[34] Y. Takamura, R. Nakane, and S. Sagahara, J. Appl. Phys. 9, 09B111 (2010).

[35] J. Zegenhagen and C. Kunz, Nucl. Instrum. Methods Phys. Res., Sect. A: Accelerators, Spectrometers, Detectors and Associated Equipment 547, v (2005).

[36] G. H. Fecher, B. Balke, A. Gloskowskii, S. Ouardi, C. Felser, T. Ishikawa, M. Yamamoto, Y. Yamashita, H. Yoshikawa, S. Ueda, and K. Kobayashi, Appl. Phys. Lett. 92, 193513 (2008).

[37] S. Ouardi, G. H. Fecher, X. Kozina, G. Stryganyuk, B. Balke, C. Felser, E. Ikenaga, T. Sugiyama, N. Kawamura, M. Suzuki, and K. Kobayashi, Phys. Rev. Lett. 107, 036402 (2011).

[38] J. Smith, Physica 24, 39 (1958).

[39] L. Berger, Phys. Rev. B. 2, 4559 (1970). 